\title{
Are Testosterone and BCL6 Critical Players in Cisplatin-Induced Nephrotoxicity in Rats?
}

\section{Amr Ahmed EL-Arabey}

Department of Pharmacology and Toxicology, University of Al-Azhar, Cairo, Egypt

"Corresponding author: Amr Ahmed EL-Arabey, Department of Pharmacology and Toxicology, Faculty of Pharmacy, University of Al-Azhar, Cairo, Egypt, Tel: 00201006180922; E-mail: ph.amrcapa@gmail.com

Received Date: June 27, 2018; Accepted Date: July 16, 2018; Published Date: July 19, 2018

Copyright: (c) 2018 El-Arabey AA. This is an open-access article distributed under the terms of the Creative Commons Attribution License, which permits unrestricted use, distribution, and reproduction in any medium, provided the original author and source are credited.

Keywords: Cisplatin; Nephrotoxicity; Gender; Testosterone; BCL6; Rats

\section{Short Communication}

Cisplatin (CP) is a powerful and dynamic cytostatic drug whose clinical application is limited by its nephrotoxicity. Although CP nephrotoxicity in patients can be monitored through diuretics and hydration regimens, the prevalence of $\mathrm{CP}$ nephrotoxicity still occurs in approximately one-third of patients undergoing $\mathrm{CP}$ treatment. Experimental animal studies have demonstrated that $\mathrm{CP}$ accumulates in the kidney more than other organs and specifically damages the proximal tubules. Human organic cation transporter 2 (hOCT2) is mainly expressed in the basolateral membrane of renal proximal tubule cells [1]. Several in-vitro and in-vivo studies from 2005 to 2008 confirmed that CP uptake via hOCT2 and rat OCT2 [2-5]. The treatment of wild type animals with $\mathrm{CP}$ and cimetidine, a substrate for OCT2 transport, reduced the nephrotoxic effects of CP [6]. Importantly, the administration of cimetidine in patients treated with $\mathrm{CP}$ produces nephron-protection effects without compromising the efficacy of CP tumor therapy [7]. Furthermore, another study revealed that hOCT2 genetic polymorphisms in combination with cimetidine protected patients from CP-induced nephrotoxicity [8]. Thus, OCT2 is a critical transporter for $\mathrm{CP}$ in the kidney and a promising target to halt the undesirable nephrotoxic effects of CP. The study by Sprowl et al. elucidated that Oct $1 / 2^{(-1-)}$ mice were not completely protected from CP-induced tubular necrosis, while the loss of tumor protein p53 in Oct $1 / 2^{(--)}$mice conferred complete protection. Clinically, noncompetitive inhibitors of OCT2 function and p53 inhibitors such as pifithrin- $\alpha$ abrogated the activation of p53 and cyclin-dependent kinase inhibitor p21 as well as the cleavage of caspase-3 in CP-treated mice [9]. In addition, another study by Wei et al. (2007) showed that the degree of renoprotection conferred by pifithrin- $\alpha$ is significantly higher than that in $\mathrm{p} 53^{(-/-)}$mice receiving cisplatin alone [10]. A careful analysis of the results of two previous studies by Sprowl et al. illustrated that pifithrin- $\alpha$ may be a unique and independent dual OCT2 and p53-inhibitor capable of circumventing the nephrotoxic effects of CP. Interestingly, the abrogation of OCT2 function through the inactivation of the 553 pathway is necessary to offer complete protection from $\mathrm{CP}$-induced nephrotoxicity. Moreover, p53 plays an important role in CP-induced nephrotoxicity independent of OCT2 function [11,12]. In 2013, a study by Nematbakhsh et al. reported that $\mathrm{CP}$-induced nephrotoxicity is gender dependent; they observed a greater intensity of damage in males than in females through an unknown mechanism [13]. Previously, I suggested this difference may be related to CP uptake by OCT2 because OCT2 renal expression is significantly higher in males than in females. Hence, $\mathrm{CP}$ uptake by the kidneys was increased through the over-expression of OCT2 in male rats and was associated with increased CP-induced nephrotoxic effects [14]. Furthermore, I proposed that the main player in the gender differences in OCT2 gene expression in rats is testosterone. This proposal is consistent with the findings of a study that found OCT2 levels to be significantly reduced in mice after castration and another study that indicated that $\mathrm{CP}$ therapy should be avoided when serum testosterone levels are high because high levels of testosterone promote CP-induced kidney injury [15]. However, several studies have exhibited contrasting effects of certain natural products or synthetic compounds on CP-induced nephrotoxicity in both sexes by using the same doses and regimens [16-21]. Substantially, a recent study concluded that formononetin, the main compound of herbal isoflavone found in the red clover plant, decreases CP accumulation in the kidney through the down regulation of OCT2 expression. Functionally, several studies have proven that formononetin is a testosterone 5 alpha-reductase inhibitor. Testosterone is reduced at the 5 alpha position to its active metabolite, dihydrotestosterone (DHT), by 5 alpha-reductase. Therefore, I recently claimed that formononetin reduced the expression of OCT2 through the DHT pathway [22]. A recent pioneer work by $\mathrm{Hu}$ et al. (2017) revealed that organic anion transporter 1/3 (OAT1/OAT3) is a novel pathway for mercapturic acid metabolite of CP uptake independent of the OCT2 pathway. The existence of a mercapturic acid metabolite of CP called NAC-1, which acts as a progenitor for renal injury through upstream of p53 [23]. The actions of these two transporters can be abrogated by nilotinib, a tyrosine kinase inhibitor without compromising the antitumor effects of CP [23]. Hence, the regulation of these renal transporters represents a new target for treatment of acute renal toxicity. In rats, the maledominant expression of Oat1/Oat3 is regulated by the transcription factor B Cell CLL/Lymphoma 6 (BCL6) and not affected by testosterone [24]. However, the implication of Oat1/Oat3 in the gender regulation of CP-induced nephrotoxicity in rats remains unanswered. Moreover, the possible regulatory function of BCL6 on gender differences of CP nephrotoxicity still uncovered also (Figure 1). Therefore, further work on the gender regulation of CP uptake by the kidney could reveal new target pathways to improve the current stand of therapeutic index of CP and offer more alternatives for clinicians and patients to promote longer survival and better quality of life. 
Citation: El-Arabey AA (2018) Are Testosterone and BCL6 Critical Players in Cisplatin-Induced Nephrotoxicity in Rats?. J Nephrol Ther 8: 315. doi:10.4172/2161-0959.1000315

Page 2 of 3

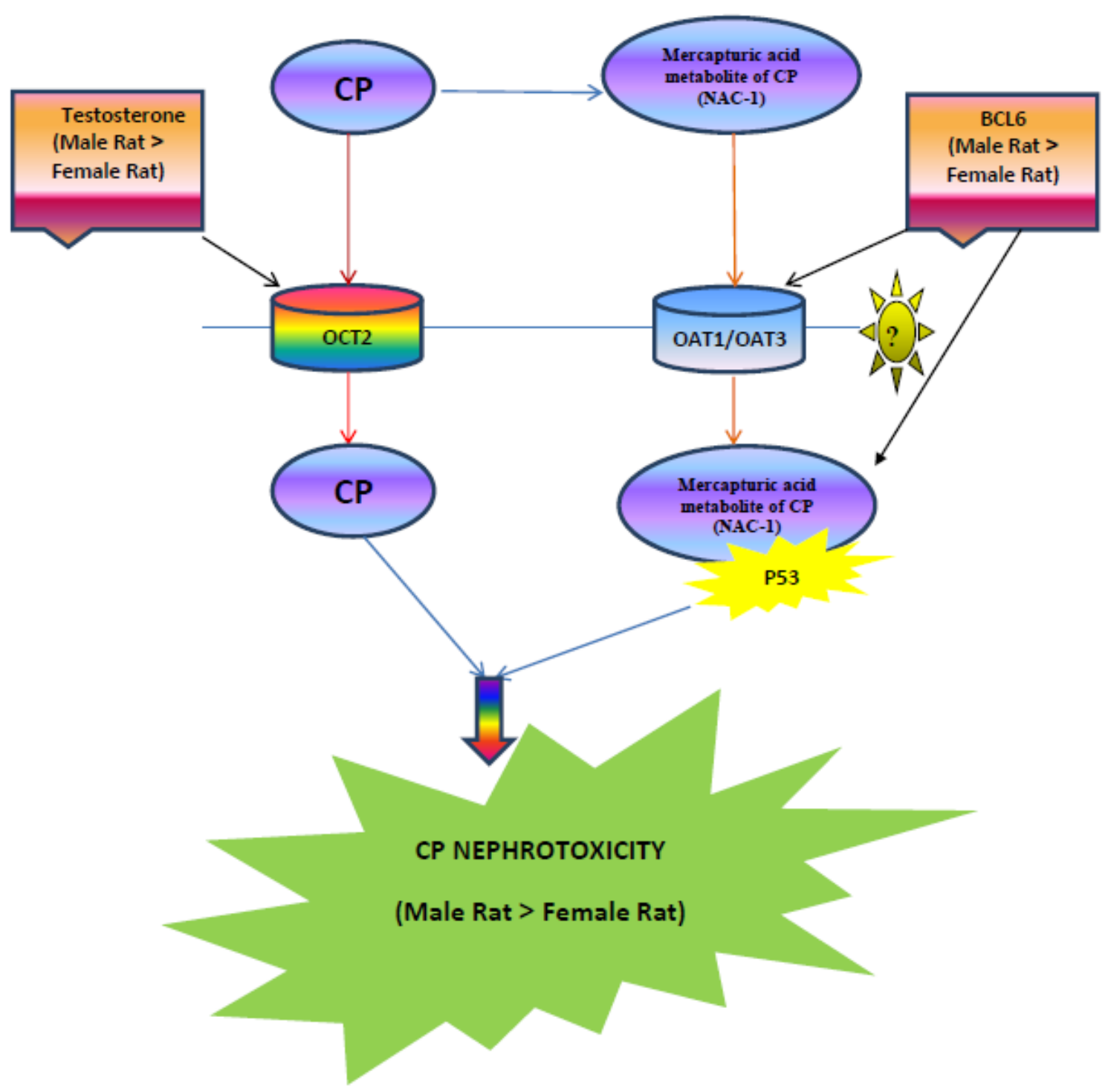

Figure 1: Testosterone is critical player in CP-induced nephrotoxicity through regulation of the OCT2. However, the role of BCL6 in kidney uptake for metabolite of $\mathrm{CP}$ remains unanswered.

\section{References}

1. Ciarimboli G (2008) Organic cation transporters. Xenobiotica 38: 936-971.

2. Filipski KK, Loos WJ, Verweij J, Sparreboom A (2008) Interaction of Cisplatin with the human organic cation transporter 2. Clin Cancer Res 14: 3875-3880.

3. El-Arabey AA and Mohnad Abdalla (2017) New insight of OCT2 Regulation as Mediator for Cisplatin- Induced Nephrotoxicity. Asian Pac J Cancer Prev 18: 1459-1460.

4. EL-Arabey AA and Salama AS (2015) Are mice and rats good experimental models to explore novel compounds against Cisplatin induced nephrotoxicity? Int J Nephrol Kidney Failure 1: 1-2.

5. EL-Arabey A A and Abd-Allah AR (2015) Antidepressants as a New Approach for Protective Interventions of Cisplatin-Induced Nephrotoxicity. J Kidney 1: 1-2.
6. Ciarimboli G, Deuster D, Knief A, Sperling M, Holtkamp M, et al. (2010) Organic cation transporter 2 mediates cisplatin-induced oto- and nephrotoxicity and is a target for protective interventions. Am J Pathol 176: 1169-1180.

7. Sleijfer DT, Offerman JJ, Mulder NH, Verweij M, van der Hem GK, et al. (1987) The protective potential of the combination of verapamil and cimetidine on cisplatin-induced nephrotoxicity in man. Cancer 60: 2823-2828.

8. Zhang J and Zhou W (2012) Ameliorative effects of SLC22A2 gene polymorphism $808 \mathrm{G} / \mathrm{T}$ and cimetidine on cisplatin-induced nephrotoxicity in Chinese cancer patients. Food Chem Toxicol 50: 2289-2293.

9. Sprowl JA, Lancaster CS, Pabla N, Hermann E, Kosloske AM, et al. (2014) Cisplatin-induced renal injury is independently mediated by OCT2 and p53. Clin Cancer Res 20: 4026-4035. 
Citation: El-Arabey AA (2018) Are Testosterone and BCL6 Critical Players in Cisplatin-Induced Nephrotoxicity in Rats?. J Nephrol Ther 8: 315. doi:10.4172/2161-0959.1000315

Page 3 of 3

10. Wei Q, Dong G, Yang T, Megyesi J, Price PM, et al. (2007) Activation and involvement of p53 in cisplatin-induced nephrotoxicity. Am J Physiol Renal Physiol 293: 1282-1291.

11. Sprowl JA, Lancaster CS, Pabla N, Hermann E, Kosloske AM, et al. (2014) Cisplatin-induced renal injury is independently mediated by OCT2 and p53. Clin Cancer Res 20: 4026-4035.

12. EL-Arabey AA (2018) An Integrative View of Cisplatin Uptake by Kidney and Renal Toxicity. J Kidney 4: 1-2.

13. Nematbakhsh M, Ebrahimian S, Tooyserkani M, Eshraghi-Jazi F, Talebi A, et al. (2013) Gender difference in Cisplatin-induced nephrotoxicity in a rat model: greater intensity of damage in male than female. Nephrourol Mon 5: 818-821.

14. EL-Arabey AA (2015) Gender difference in cisplatin-induced nephrotoxicity in a rat model. Nephrourol Mon 7: e23595.

15. El-Arabey AA (2015) Sex and age differences related to renal OCT2 gene expression in cisplatin-induced nephrotoxicity. Iran J Kidney Dis 9: 335-336.

16. EL-Arabey AA (2015) Sex Differences in Protective Effect of Recombinant Human Erythropoietin Against Cisplatin-induced Nephrotoxicity in Rats. Iran J Kidney Dis 9: 163.

17. EL-Arabey AA (2015) Comment on: Pomegranate flower extract does not prevent cisplatin-induced nephrotoxicity in female rats. Int J Prev Med 6: 47.
18. EL-Arabey AA (2016) Comment on: Effect of pomegranate flower extract on cisplatin-induced nephrotoxicity in male rats. Int J Prev Med 7: 9.

19. El-Arabey AA (2016) Negative Response of Phytoestrogens of Pomegranate Flower Extract against Cisplatin-induced Nephrotoxicity in Female Rats. Int J Prev Med 7: 89.

20. El-Arabey AA (2015) Is testosterone responsible for age differences in the susceptibility of cisplatin-induced nephrotoxicity in male C57BL/6 mice? Clin Pract 13: 1-2.

21. El-Arabey AA (2016) Dual function of OCT2 and MATE1 in cisplatin induced nephrotoxicity. Pharmacol Res 119: 493.

22. El-Arabey AA (2017) Dihydrotestosterone as mediator for cisplatin induced nephrotoxicity: New insight down the road. Toxicology 387: 108.

23. Hu S, Leblanc AF, Gibson AA, Hong KW, Kim JY, et al. (2017) Identification of OAT1/OAT3 as Contributors to Cisplatin Toxicity. Clin Transl Sci 10: 412-420.

24. Wegner W, Burckhardt BC, Burckhardt G, Henjakovic M (2012) Maledominant activation of rat renal organic anion transporter 1 (Oat1) and 3 (Oat3) expression by transcription factor BCL6. PLoS One 7: e35556. 